\title{
ИНДИВИДУАЛЬНЫЕ ОСОБЕННОСТИ ЧЕЛОВЕКА - ОБЪЕКТА ВОЗДЕЙСТВИЯ КАК ФАКТОР ЭФФЕКТИВНОСТИ ПСИХОЛОГИЧЕСКОГО ВОЗДЕЙСТВИЯ
}

\section{INDIVIDUAL CHARACTERISTICS \\ OF A PERSON - AN OBJECT \\ OF INFLUENCE AS A FACTOR \\ OF THE EFFECTIVENESS \\ OF PSYCHOLOGICAL INFLUENCE}

\section{Latynov}

Summary: The article discusses the issues of personalized influence influence that takes into account the individual psychological characteristics of the people to whom it is directed. Eight groups of significant in this respect characteristics (bases of personalization) have been identified: the psychological state of a person, features of his goals and motivation, cognitive characteristics, features of the structure of attitudes, social identity, cultural characteristics, personality traits, subjective vulnerability to tactics of influence. The effectiveness of personalization increases when it is aimed at several characteristics of a person - the object of influence, and he himself uses a systematic analysis of information. Under certain conditions (the use of a stigmatized identity as the basis for personalization, the recipient's perception of personalization as a violation of privacy or an attempt to manipulate), personalization reduces the effectiveness of the impact.

Keywords: psychological influence, personalized influence, influence effectiveness, object of influence, systematic analysis of information.
$\mathrm{P}$ астущий массив данных, свидетельствующих о значимости индивидуальных особенностей человека объекта воздействия как фактора эффективности психологического воздействия, обусловил повышенное внимание ученых к персонализированному воздействию [16; 25; 31]. В случае такого рода воздействия предполагается учет самых разных индивидуальных особенностей человека, на которого оно направлено: имени, пола, этнической принадлежности, психологического состояния, мотивации, ценностей, личностных черт и т.д.

Обращение к теме персонализированного воздействия обусловлено как логикой развития психологического знания, так и запросами общественной практики: прежде всего, экономики, политики и здравоохранения. Развитие новых средств коммуникации открывает дополнительные возможности индивидуализации воздействия на человека, что повышает востребованность научных данных о закономерностях персонализирован-

\author{
Латынов Владислав Викторович \\ К.nсх.Н., С.Н.С., ФГБУН Институт психологии Российской \\ Академии Наук (Москва) \\ vladlat5@lenta.ru
}

Аннотация: В статье обсуждаются вопросы персонализированного воздействия, - воздействия, учитывающего индивидуально-психологические особенности людей, на которых оно направлено. Выделено восемь групп значимых в данном отношении характеристик (оснований персонализации): психологическое состояние человека, особенности его целей и мотивации, когнитивные характеристики, особенности структуры аттитюдов, социальная идентичность, культурные особенности, черты личности, субъективная уязвимость к тактикам воздействия. Эффективность персонализации возрастает в том случае, когда она нацелена на несколько характеристик человека - объекта воздействия, а он сам использует систематический анализ информации. При определенных условиях (использование в качестве основания персонализации стигматизированной идентичности, восприятие реципиентом персонализации как нарушение приватности или попытки манипулирования) персонализация вызывает снижение эффективности в0здействия.

Ключевые слова: психологическое воздействие, персонализированное воздействие, эффективность воздействия, объект воздействия, систематический анализ информации.

ного воздействия.

Неоднократно было продемонстрировано, что персонализированное воздействие способно ощутимо изменять мнения, аттитюды и убеждения людей. Так, персонализация сообщений в Фейсбуке положительно влияла на идентификацию потребителей с брендом и намерение его использовать [32]. В работе С. Матз с соавт. (2017) обнаружено увеличение эффективности воздействия при совпадении характера используемых приемов воздействия и личностных особенностей лица, на которого они направлены [26]. Например, в том случае, когда экстраверты получали сообщение, текст и визуальный ряд которого, наиболее соответствовал, по мнению учёных, этому личностному типу. Эффективность персонализации объясняется тем, что человек - объект воздействия в той или иной степени осознает близость сообщения своим особенностям и позитивно на это реагирует [10].

Рассматривая ситуацию психологического воздей- 
ствия, можно выделить в ней четыре основных элемента: субъект и объект воздействия, средство воздействия и его контекст [1]. Соответственно персонализация воздействия может осуществляться в трех направлениях: субъект-объект, контекст-объект, средство-объект. В первом случае для повышения эффективности воздействия выбирается такой его субъект, который соответствует тем или иным особенностям лица, на которое воздействие направлено (полу, расе, эмоциональному состоянию, черте личности и др.) [13]. Например, воздействие на потребителей с высоким эмоциональным интеллектом оказывалось более эффективным в том случае, когда оно осуществлялось субъектом с аналогичной степенью его выраженности [21].

Второе направление персонализации (контекст-ориентированное) предполагает модификацию некоторых элементов контекста воздействия для их большего соответствия характеристикам человека, на которого оно направлено [14]. Например, проигрывание немецкой музыки в магазине приводило к росту продаж немецкого вина, а французской - французского посредством активации соответствующей национальной идентичности (немецкой в первом случае, а французской — во втором) [27].

Следует отметить, что два вышеуказанных направления персонализации исследованы относительно слабо, возможно в связи с тем, что в реальности довольно трудно изменить субъекта и контекст воздействия. Гораздо большее внимание ученых привлекает персонализация средств воздействия. Это обусловлено тем, что задача изменения характеристик средств воздействия (например, коррекция текста убеждающего сообщения) довольно легко реализуема на практике. К настоящему времени изучен широкий спектр особенностей человека - объекта воздействия, «подстраивание» под которых способствует повышению его эффективности. Можно выделить восемь групп значимых в данном отношении характеристик (оснований персонализации): психологическое состояние человека, особенности его целей и мотивации, когнитивные характеристики, особенности структуры аттитюдов, социальная идентичность, культурные особенности, черты личности, субъективная уязвимость к тактикам воздействия.

Рассмотрим последовательно указанные группы. Прежде всего, для персонализации воздействия можно использовать психологическое состояние человека [37]. Причем речь идет как о количественной стороне данного явления (уровень психологического возбуждения), так и о качественной (общий модус настроения: позитивный или негативный, конкретные эмоции, переживаемые человеком). Было обнаружено, что акцент на позитивных аспектах покупки некоторого товара (в сравнении с подчеркиванием «минусов», вызванных его непокупкой) оказывается более эффективным приемом воздействия на лиц в позитивном эмоциональном состоянии [4]. Верно и обратное: указание на негативные стороны отказа от покупки эффективнее действовало на людей, находящихся в плохом настроении.

Используются для персонализации и когнитивные особенности людей. Речь идет о так называемых майндсетах - установках на определенный вид познания мира (абстрактность-конкретность мышления, установка на творчество и др.). Учет данных особенностей при осуществлении воздействия повышает его эффективность [12].

Еще одним основанием персонализации воздействия являются цели и особенности мотивации человека. Например, в психологии принято различать гедонистические (получение удовольствия) и утилитарные (получение выгоды) цели. Существуют индивидуальные различия в данном отношении: одни люди более склонны к достижению гедонистических целей, а другие - утилитарных [2]. Подобные межиндивидуальные различия используются для повышения эффективности воздействия. Установлено, что на «гедонистов» эффективнее действовали рекламные сообщения, подчеркивающие эмоциональные, связанные с чувством удовольствия аспекты потребления некоторого продукта, а на «утилитаристов» - акцентирующие полезность и выгодность обладания продуктом [23].

«Подстраивать» убеждающие сообщения можно и под более общие мотивационные образования людей (мотивация достижения (т. е. ориентация на успех) и мотивация избегания (боязнь неудачи)) [11]. Обнаружено, что на людей с мотивацией достижения сильнее действовали убеждающие сообщения, подчеркивающие позитивный эффект от действий человека («на пожертвованные вами средства библиотека университета купит новые книги»), а на «избегающих» - сообщения, указывающие на негативные эффекты («без благотворительной помощи библиотеке придется выбросить часть книг») [15].

Для персонализации используются также особенности структуры аттитюдов человека. Как известно, аттитюды могут различаться не только валентностью (позитивный-негативный), но и своими основаниями (эмоции или когниции), функциями, которые они выполняют, взаимосвязью с моралью и политическими убеждениями. Например, апелляция к эмоциям успешнее изменяла аттитюды, основанные на эмоциях, а логическое убеждение - аттитюды с когнитивным основанием [20]. Аналогичным образом воздействие оказывается более эффективным при соответствии аргументов убеждающего сообщения моральным основаниям человека и функциональным особенностям его аттитюдов [17; 25]. 
Основанием персонализации может выступать социальная идентичность человека. Как показали исследования, акцент на идентичности болельщика [28] и классовой идентичности [35] людей позволил с успехом изменять их аттитюды. Возможен при персонализации и учет культурных особенностей людей [29]. Например, есть данные о том, представителям Запада более свойственна аналитичность мышления, а представителям восточной культуры - целостность [31]. Эти различия были использованы для персонализации: эффект воздействия возрастал в тех случаях, когда «аналитикам» предъявлялся рекламируемый товар в изоляции, а «холистам» - в более широком контексте [24].

Популярным направлением в последние годы стала персонализация воздействия, основанная на личностных чертах человека. Анализ широкого спектра черт (экстраверсии, нейротизма, открытости опыту и др.) показал, что «подстраивание» убеждающих сообщений к соответствующей черте позитивно влияло на эффективность воздействия [36; 38].

Персонализация может осуществляться и в отношении наиболее предпочитаемых человеком стратегий воздействия. Так, М. Каптейн с соавт., собрав информацию об индивидуальной подверженности респондентов различным стратегиям воздействия (авторитет, дефицит, социальное доказательство, последовательность), затем использовали ее для успешного изменения как их аттитюдов, так и поведения [18; 19].

Эффективность персонализации обусловлена действием разнообразных психологических механизмов. В самых типичных ситуациях воздействия, когда у человека нет ни особого желания к систематической обработке сообщений, ни направленности на периферические ключевые стимулы, персонализированные сообщения способствуют более внимательному и тщательному анализу поступающей информации [7]. Было обнаружено, что персонализированное сообщение, содержащее «сильные» аргументы, более успешно изменяло аттитюды людей в сравнении с неперсонализированным сообщением, включающим те же аргументы [34]. Именно при наличии углубленного анализа человек способен разобраться в том, насколько убедительны, серьезны аргументы, с помощью которых его побуждают принять некоторую точку зрения. Если же подобная когнитивная установка отсутствует и человек стремится минимизировать свои размышления над приходящими сообщениями, полагаясь на простейшие эвристики, то действенность сильных и слабых аргументов оказывается примерно равной.

Ученые выяснили условия, при которых эффект от персонализации воздействия оказывается наиболее выраженным и стойким. Согласно современным представ- лениям о психологическом воздействии «сильные» (т.е. долговечные, устойчивые к попыткам их изменения и ощутимо влияющие на поведение) аттитюды возникают тогда, когда они формируются на основании систематического анализа информации (по сравнению с использованием эвристик) [1]. В случае подобного анализа человек подробно и внимательно рассматривает различные аспекты поступающих к нему сообщений.

Исследования подтвердили действие указанной закономерности и в случае персонализированного воздействия. В работе Пьеро с соавт. (2012) участники с различной мотивацией к систематической обработке информации получали соответствующие их когнитивной установке убеждающие сообщения: лица с высокой мотивацией получали длинные сообщения, а лица с низкой - короткие [30]. Эффект воздействия этих сообщений фиксировался сразу после эксперимента и через три недели после его проведения. Было обнаружено, что кратковременное изменение аттитюдов вызывали как короткие, так и длинные сообщения, однако долговременное (сохранившееся в течение трех недель) изменение аттитюдов имело место только в результате воздействия длинных, т.е. подвергавшихся углубленному, систематическому анализу сообщений.

Рассмотрим способы увеличения эффективности персонализированного воздействия. Поскольку, как мы уже отмечали, изменение аттитюдов и убеждений, достигаемое посредством углубленного анализа, оказывается наиболее стойким и долговечным, то для повышения эффективности персонализации необходимо побудить человека «хорошенько» подумать над поступившим к нему убеждающим сообщением [31]. Еще одним способом роста ее успешности является воздействие на те аттитюды и убеждения людей, которые для них наиболее значимы. Если удается добиться такого рода изменений, то эффект от персонализированного воздействия сохраняется надолго [9]. Таргетирование одновременно нескольких характеристик человека - объекта воздействия также делает персонализацию более эффективной [16].

Несмотря на то, что персонализация воздействия в большинстве случаях приводит к росту его эффективности, однако при определенных условиях она вызывает обратный эффект. Например, подобное случается в тех случаях, когда человек считает, что с помощью персонализации покушаются на его приватность [33]. Так, люди, увидев рекламу книжного магазина, соответствующую их предыдущей истории просмотров в Интернете, в зависимости от отношения к подобной практике использования их данных реагировали на это сообщение по-разному. Те, кто считали, что сбор информации для такого варианта персонализации был нелегитимен, отнеслись к рекламному сообщению более негативно по сравнению с теми, кто не видел в этом нарушения при- 
ватности [22].

Негативный эффект от персонализации встречается и тогда, когда убеждающее сообщение оказывается нацелено на стигматизированную идентичность человека, что заставляет его чувствовать себя несправедливо оцененным или стереотипно воспринимаемым [8]. Например, такой эффект возникал в тех случаях, когда имеющие избыточный вес потребители считали, что получили информацию о программе похудания именно из-за подобной физической особенности. В свою очередь, это побуждало их генерировать больше негативных мыслей о поступившем сообщении и приводило к ослаблению намерения вести здоровый образ жизни и бороться с избыточным весом [6]. Эффект от персонализации снижается и в тех ситуациях, когда человек начинает считать обращенное к нему убеждающее сообщение манипуля- цией и угрозой своей свободе выбора, что вызывает негативные эмоции и приводит к отвержению транслируемой сообщением точки зрения [3; 5].

Современные исследования показали, что на эффективность психологического воздействия ощутимое влияние оказывают индивидуально-психологические особенности людей - объектов воздействия. Основаниями персонализации могут выступать самые разные психологические характеристики (психологическое состояние человека, особенности его целей и мотивации, черты личности и т.д.). Это открывает дополнительные возможности для активного использования персонализированного воздействия при решении прикладных задач во многих сферах социальной жизни (политике, экономике, медицине и др.).

\section{ЛИТЕРАТУРА}

1. Латынов В.В. Психология коммуникативного воздействия. М., 2013.

2. Abelson, R.P., Prentice, D.A. Beliefs as possessions: A functional perspective // Attitude structure and function. A.R. Pratkanis, S.J. Breckler, A.G. Greenwald (Eds.). Psychology Press. 1989. P. 361-379.

3. Brinol, P., Rucker, D.D., Petty, R.E. Naive theories about persuasion: Implication for information processing and consumer attitude change // International Journal of Advertising. 2015. V. 34. P. 85-106.

4. Cho, H., Choi, J. Predictors and the role of attitude toward the message and perceived message quality in gain- and loss-frame antidrug persuasion of adolescents // Health Communication. 2010. V. 25. P. 303-311.

5. David, P., Henry, A., Srivastava, J., Orcena, J., Thrush, J. Reactance to a tailored multimedia intervention encouraging teachers to promote cover-the-cough // Journal of Health Communication. 2012. V. 17. P. 915-928.

6. Derricks, V., Earl, A. Information Targeting Increases the Weight of Stigma: Leveraging Relevance Backfires When People Feel Judged // Journal of Experimental Social Psychology. 2019. V. 82. P. 277-293.

7. Dimmock, J.A., Jackson, B., Clear, S.E., Law, K.H. Matching temporal frame to recipients' time orientation in exercise messaging: Does argument quality matter? // Psychology of Sport and Exercise. 2013. V. 14. P. 804-812.

8. El Hazzouri, M., Hamilton, L.K. Why us?! How members of minority groups react to public health advertisements featuring their own group // Journal of Public Policy and Marketing. 2019. V. 38. P. 372-390.

9. Evans, L.M., Petty, R.E. Self-guide framing and persuasion: Responsibly increasing message processing to ideal levels // Personality and Social Psychology Bulletin. 2003. V. 29. P. 313-324.

10. Gawronski, B., Bodenhausen, G.V., Becker, A.P. I like it, because I like myself: Associative selfanchoring and post-decisional change of implicit evaluations // Journal of Experimental Social Psychology. 2007. V. 43. P. 221-232.

11. Gerend, M.A., Shepherd, J.E. Using message framing to promote acceptance of the human papillomavirus vaccine // Health Psychology. 2007. V. 26. P. 745-752.

12. Han, D., Duhachek, A., Agrawal, N. Coping and construal level matching drives health message effectiveness via response efficacy or self-efficacy enhancement // Journal of Consumer Research. 2016. V. 43. P. 429-447.

13. Hartman, T.K., Weber, C.R. Who said what? The effects of source cues in issue frames // Political Behavior. 2009. V. 31. P. 537-558.

14. Hunter, R., Mukerji, B. The role of atmospherics in influencing consumer behaviour in the online environment // International Journal of Business and Social Science. 2011. V. 2. P. 118-125.

15. Jeong, E.S., Shi, Y., Baazova, A., Chiu, C., Nahai, A., Moons, W.G., Taylor, S.E. The relation of approach/avoidance motivation and message framing to the effectiveness of persuasive appeals // Social Influence. 2011. V. 6. P. 15-21.

16. Joyal-Desmarais, K., Rothman, A.J., Snyder, M. How do we optimize message matching interventions? Identifying matching thresholds, and simultaneously matching to multiple characteristics // European Journal of Social Psychology. 2020. V. 50. P. 701-720.

17. Julka, D.L., Marsh, K.L. An attitude functions approach to increasing organ-donation participation // Journal of Applied Social Psychology. 2005. V. 35. P. 821-849.

18. Kaptein, M., Markopoulos, P., de Ruyter, B., Aarts, E. Personalizing persuasive technologies: Explicit and implicit personalization using persuasion profiles // International Journal of Human-Computer Studies. 2015. V. 77. P. 38-51.

19. Kaptein, M.C., de Ruyter, B., Markopoulos, P., Aarts, E. Tailored persuasive text messages to reduce snacking // ACM Transactions on Interactive Intelligent Systems. 2012. V. 2. P. $10-35$. 
20. Keer, M., van den Putte, B., de Wit, J., Neijens, P. The effects of integrating instrumental and affective arguments in rhetorical and testimonial health messages // Journal of Health Communication. 2013. V. 18. P. 1148-1161.

21. Kidwell, B., Lopez-Kidwell, V., Blocker, C., Mas, E. Birds of a feather feel together: Emotional ability similarity in consumer interactions // Journal of Consumer Research. 2020. V. 47. P. 215-236.

22. Kim, T., Barasz, K., John, L.K. Why am I seeing this ad? The effect of ad transparency on ad effectiveness // Journal of Consumer Research. 2019. V. 45. P. 906-932.

23. Klein, K., Melnyk, V. Speaking to the mind or the heart: Effects of matching hedonic versus utilitarian arguments and products // Marketing Letters. 2014. V. 27. P. 131-142.

24. Liang, B., Runyan, R.C., Fu, W. The effect of culture on the context of ad pictures and ad persuasion: The role of context-dependent and context-independent thinking // International Marketing Review. 2011. V. 28. P. 412-434.

25. Luttrell A., Philipp-Muller A.Z., Petty R.E. Challenging moral attitudes with moral messages // Psychological Science. 2019. V. 30. P. 1136-1150.

26. Matz, S.C., Kosinski, M., Nave, G. Stillwell, D.J. Psychological targeting as an efective approach to digital mass persuasion // Proceedings of the National Academy of Sciences. 2017. V. 114 (48). P. 12714-12719.

27. North, A.C., Hargreaves, D.J., McKendrick, J. The influence of in-store music on wine selections // Journal of Applied Psychology. 1999. V. 84. P. 271-276.

28. Ottati, V., Rhoads, S., Graesser, A.C. The effect of metaphor on processing style in a persuasion task: A motivational resonance model // Journal of Personality and Social Psychology. 1999. V. 77. P. 688-697.

29. Orji, R. Persuasion and Culture: Individualism-Collectivism and Susceptibility to Influence Strategies // International Workshop on Personalization in Persuasive Technology (PPT'16). Salzburg. 2016. P. 30-39.

30. Pierro, A., Mannetti, L., Kruglanski, A.W., Klein, K., Orehek, E. Persistence of attitude change and attitude-behavior correspondence based on extensive processing of source information // European Journal of Social Psychology. 2012. V. 42. P. 103-111.

31. Teeny, J.D., Siev, J., Brinol, P., Petty, R.E. A review and conceptual framework for understanding personalized matching effects in persuasion // Journal of Consumer Psychology. 2021. V. P. 382-414.

32. Tran, T.P., Lin, C-W., Baalbaki, S., Guzmán, F. How personalized advertising affects equity of brands advertised on Facebook? A mediation mechanism // Journal of Business Research. 2020. V. 120. P. 1-15.

33. Van Doorn, J., Hoekstra, J. C. Customization of online advertising: The role of intrusiveness // Marketing Letters. 2013. V. 24. P. 339-351.

34. Wan, E.W., Rucker, D.D. Confidence and construal framing: When confidence increases versus decreases information processing // Journal of Consumer Research. 2013. V. 39. P. 977-992.

35. Whillans, A.V., Caruso, E.M., Dunn, E.W. Both selfishness and selflessness start with the self: How wealth shapes responses to charitable appeals // Journal of Experimental Social Psychology. 2017. V. 70. P. 242-250.

36. Winter, S., Maslowska, E. Vos, A.L. The efects of trait-based personalization in social media advertising // Computer in Human Behavior. 2021. V. 114. 106525. URL: https://doi.org/10.1016/j.chb.2020.106525 (дата обращения: 30.11.2021).

37. Yan, L., Liu, M.T., Chen, X., Shi, G. An arousal-based explanation of affect dynamics // European Journal of Marketing. 2016. V. 50. P. 1159-1184.

38. Zarouali, B., Dobber, T., De Pauw, G., de Vreese, C.. 2020. Using a Personality-Profiling Algorithm to Investigate Political Microtargeting: Assessing the Persuasion Effects of Personality-Tailored Ads on Social Media // Communication Research. 2020. URL: https://doi.org/10.1177/0093650220961965 (дата обращения: 30.11.2021).

(с) Латынов Владислав Викторович (vladlat5@lenta.ru). 